\title{
A hybrid finite element analysis and evolutionary computation method for the design of lightweight lattice components with optimized strut diameter
}

\author{
Konstantinos Salonitis $^{\mathrm{a}^{*}}$, Dimitrios Chantzis ${ }^{\mathrm{b}}$, Vassilios Kappatos ${ }^{\mathrm{c}}$ \\ ${ }^{a}$ Manufacturing Department, Cranfield University, Cranfield, Bedfordshire MK43 0AL, UK \\ ${ }^{\mathrm{b}}$ Spectrum Engineering Solutions Ltd.,7 Ellison Road Barnes, London, SW13 0AD,UK \\ ${ }^{c}$ Department of Technology and Innovation (ITI), University of Southern Denmark (SDU), Campusvej 55, DK- \\ 5230 Odense M, Denmark
}

\begin{abstract}
* Corresponding author. Department of Manufacturing, Cranfield University, Cranfield, Bedfordshire MK43 0AL, UK Tel.: +44 (0) 1234 758347. E-mail address: k.salonitis@cranfield.ac.uk
\end{abstract}

\begin{abstract}
Components incorporating lattice structures have become very popular lately due to their lightweight nature and the flexibility that additive manufacturing offers with respect to their fabrication. However, design optimization of lattice components has been addressed so far either with empirical approaches or with the use of topology optimization methodologies. An optimization approach utilizing multi-purpose optimization algorithms has not been yet proposed. This paper presents a novel user-friendly method for the design optimization of lattice components towards weight minimization, which combines finite element analysis and evolutionary computation. The proposed method utilizes the cell homogenization technique in order to reduce the computational cost of the finite element analysis and a genetic algorithm in order to search for the most lightweight lattice configuration. A bracket consisting of both solid and lattice regions is used as a case study in order to demonstrate the validity and effectiveness of the method, with the results showing that its weight is reduced by $13.5 \%$ when using lattice structures. A discussion about the efficiency and the implications of the proposed approach is presented.
\end{abstract}

Keywords: Lattice structures; Design optimization; Genetic algorithm; Homogenization Nomenclature

\begin{tabular}{llll}
\hline $\boldsymbol{d}$ & Strut diameter & $\boldsymbol{W}$ & Weight of a fully dense cube \\
$\boldsymbol{E}$ & Young's modulus & $\boldsymbol{\varepsilon}$ & Strain
\end{tabular}




\begin{tabular}{|llll|}
\hline $\boldsymbol{F}$ & Force & $\boldsymbol{v}$ & Poisson's ratio \\
$\boldsymbol{l}$ & Edge length & $\boldsymbol{\pi}$ & pi \\
$\boldsymbol{L B}$ & Lower bound of strut diameter value & $\boldsymbol{\rho}$ & Density of a lattice cell \\
$\boldsymbol{U B}$ & Upper bound of strut diameter value & $\boldsymbol{\rho}_{\mathbf{0}}$ & Density of the bulk material \\
$\boldsymbol{w}$ & Weight of the lattice cell & $\boldsymbol{\sigma}$ & stress \\
\hline
\end{tabular}

\section{Abbreviations}

\begin{tabular}{|llll|}
\hline AM & Additive manufacturing & FEA & Finite element analysis \\
BCC & Body-centered cubic & FEM & Finite element method \\
BESO & $\begin{array}{l}\text { Bidirectional evolutionary structural } \\
\text { optimization }\end{array}$ & GA & Genetic algorithm \\
DG & Discontinuous Galerkin & LM & Levenburg-Marquardt \\
DMLS & Direct metal laser sintering & PSO & Particle swarm optimization \\
DOF & Degree of freedom & RVE & Representative volume element \\
DOS & Disk operating system & SIMP & $\begin{array}{l}\text { Solid isotropic material with } \\
\text { penalty }\end{array}$ \\
EBM & Electron beam melting & SLM & $\begin{array}{l}\text { Selective laser melting } \\
\text { Sequential quadratic }\end{array}$ \\
FDM & Fused deposition modelling & SQP & \begin{tabular}{l} 
programming \\
\hline
\end{tabular}
\end{tabular}

\section{Introduction}

The need for sustainable products and efficient use of natural resources has driven to a lightweight design trend. European Union authorities have set targets of achieving $20 \%$ energy efficiency and reducing greenhouse gas emissions by $20 \%$ until 2020, clearly shaping the picture of future European manufacturing and economy in general [1]. To tackle these challenges, the global manufacturing industry has come up with new approaches that decrease the environmental impact of the state of play practices [2]. Bionic design and topology optimization are examples of innovative design approaches that can significantly reduce the environmental impact of a part through its whole lifecycle, mainly due to weight savings, which can reach up to $40 \%$ of the non-optimized part's weight [3], [4]. However, realizing these optimized designs is at most cases constrained by the current manufacturing technologies which are often 
unable to combine product complexity with cost and resource efficiency; "most complex" still means "most expensive" and "most inefficient" from the point of view of primary resources consumption. New methods for design for additive manufacturing are developed though for enabling the reap of benefits of these methods [5].

Recent manufacturing techniques such as Powder Bed Additive Manufacturing processes allow for such innovative design approaches. One of the most applicable, is the topology optimisation, which is driven by the need to put material only where it is needed for a specific application. Topology optimised design concepts can further improve the structural performance of a component while reducing its weight. However, most of the times, the outcome of this optimisation process, is components with lattice structures which cannot be fabricated by using conventional, subtractive manufacturing processes.

Truss-like lattice structures have become an attractive option to engineers when designing lightweight metallic components as they offer a vast range of options as far as it regards design patterns, geometrical proportions, mechanical properties and suitability for specific applications. As a result, design of lattice components can become more "standardized", offering the chance for cost and time savings. In addition, lattice structures are superior to foams and honeycombs in terms of strength-to-weight ratio and also advantageous in other than structural applications (e.g. heat transfer), due to their open structure [6].

\subsection{Lattice topologies}

The arrangement and the number of struts inside a lattice cell is called lattice cell topology. This topology also defines the arrangement, called lattice truss topology, of more than one cell to form a lattice truss. Common lattice truss topologies include the octet truss, tetrahedral lattice truss, lattice block, pyramidal lattice truss and 3D kagome [7], as illustrated in Figure 1. Typical strut diameters range from 100 to 1,000 $\mu \mathrm{m}$, while the edge size for a lattice cell is in the region of 1-10 $\mathrm{mm}$ [8]. However, conventional processes, such as investment casting and metal forming, can be time and cost consuming for the manufacturing of lattice structures and also put limitations in the design freedom and geometrical complexity, mainly due to tool access constraints [9]. 


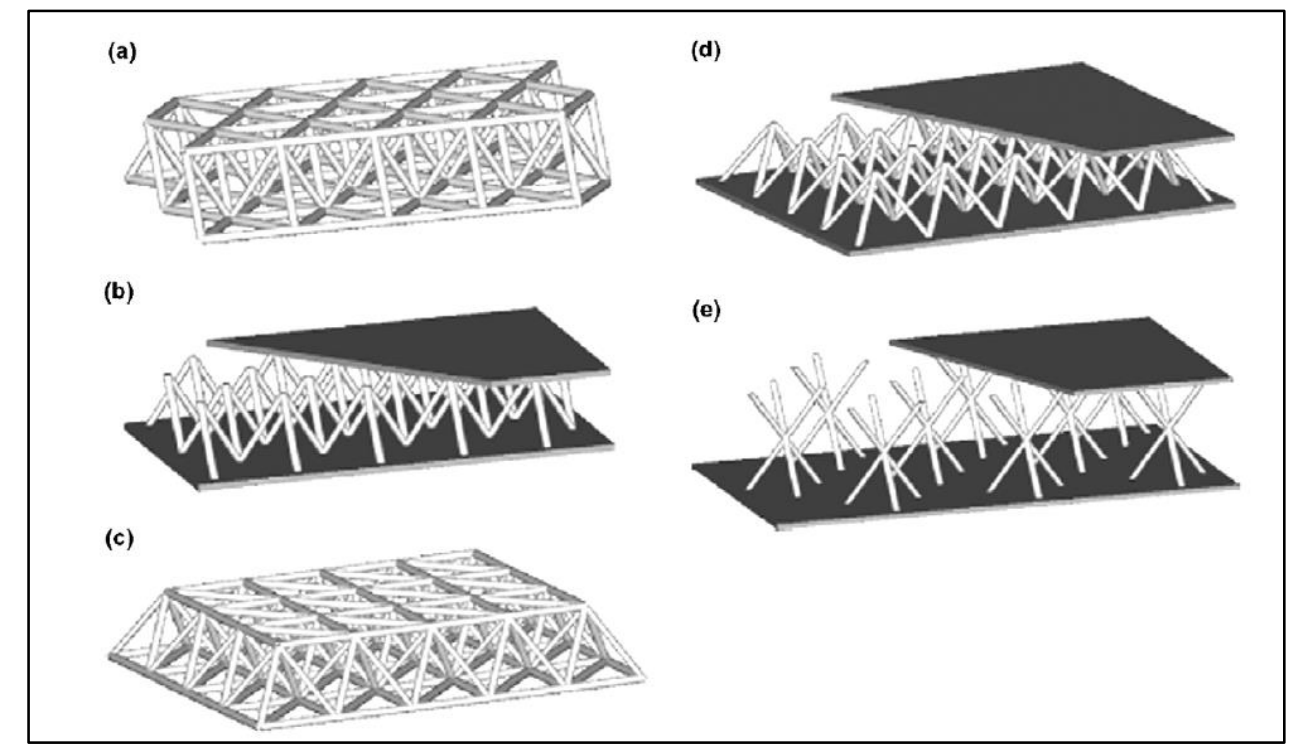

Figure 1. Common lattice truss topologies: (a) octet truss, (b) tetrahedral lattice truss, (c) lattice block, (d) pyramidal lattice truss and (e) 3D kagome [7].

\subsection{Additive manufacturing of lattice components}

The recent developments in additive manufacturing (AM) for metallic materials have allowed the fabrication of parts composed entirely or partially from lattice structures with optimized geometry and enhanced dimensional accuracy [10], [11], [12]. The aerospace and medical industries are those that have benefited the most from the ability to build lattice structures without almost any design considerations. In the medical industry, lattice structures are primarily used for the fabrication of implant scaffolds [13]. On the other hand, in the aerospace industry, lattice structures are favourable mostly for their impact and compressive loading response [14] besides their lightweight nature which is crucial for this sector. Various AM processes have been applied so far for the fabrication of lattice structures. However, as AM processes can lead to various types of defects [15], [16], the quality of the manufactured parts is of major research interest. The research focus has been on optimizing the process parameters for manufacturing AM parts (as an example D'Alvise et al. has focused in the part distortion due to residual stresses and how this could be optimized through modelling [17], [18]), with few studies on the lattice structures. Yan et al. [9] investigated the dimensional accuracy and the mechanical properties of AlSi10Mg lattice structures fabricated via direct metal laser sintering (DMLS). The mechanical properties of Ti6Al4V lattice structures, built by selective laser melting (SLM) with optimized unit cell topology, were studied by Challis et al. [19]. The experimental results showed good agreement with the results from the numerical analysis. SLM was also employed by Niendorf et al. [20] when studying the effect of the geometrical dimensions of the lattice cell to the microstructure evolution during processing. Electron 
beam melting (EBM) was utilized by Cansizoglu et al. [21] and Karlsson et al. [22] when investigating the mechanical properties of Ti6Al4V lattice structures. From the above, it can be concluded that studying the properties of lattice structures in accordance with the fabrication method is of major importance for two distinct reasons. In the first place, AM is a quite unexplored region [23] and the impact of the process parameters on the performance of the lattice structure has to be further investigated. Secondly, as the principal dimensions of a lattice cell (i.e. strut diameter, edge size) are at most cases comparable with the spot diameter of an AM process heat source and also the powder grain size, even minor dimensional inaccuracies can have a remarkable effect on the overall manufacturing quality of the lattice structure.

\subsection{Design optimization of lattice components}

As weight minimization can offer significant cost savings during the whole lifecycle of a product [3], design optimization to achieve minimum weight for lattice structures is a trending research topic. An empirical methodology for optimizing lattice structures is performing finite element analysis (FEA) in an initial geometry and then matching the number and the diameter of the lattice structure's struts with the stress field. This methodology has been applied by Nguyen et al. [24] for optimizing the design of a micro aerial vehicle fuselage. According to the researchers, this method can offer solutions close to the global optimum. The validity of this method can be further supported by the findings of Reinhart and Teufelhart [25], who investigated the effect of the diameter, number and angle of the struts on the stiffness of torqueloaded lattice shaft. It was observed that the stiffness reached its maximum value when the struts were orientated along the flux of force. Orientating the struts along the flux of force has been also proved effective for the design of lattice beams subjected to bending [26]. The optimized beam had almost the double stiffness-to-weight ratio when compared to the standard periodic-built beam. Well-established structural optimization methodologies, such as the bidirectional evolutionary structural optimization (BESO) [27] and the solid isotropic material with penalty (SIMP) method [28] have been already applied for optimization of lattice structures. Beyond empirical methodologies and structural optimization techniques, little attention has been given to the use of generic solution space search schemes in order to obtain the optimal parameters of lattice structures. Chu et al. [29] utilized both the particle swarm optimization (PSO) and the Levenburg-Marquardt (LM) algorithms in order to optimize the strut diameters in a lattice cantilever beam for achieving minimum deflection and total weight without surpassing a stress limit. Although both algorithms resulted in very good designs, the LM algorithm converged much more quickly than the PSO algorithm. However, both algorithms needed a good initial solution in order to ensure satisfying performance. Other approaches to lattice structural optimization with the use of intelligent search methods include the use of the Sequential Quadratic Programming (SQP) algorithm [30] and the Conlin algorithm [31]. In addition to part design, optimization can be also 
performed at the lattice cell level in order to explore new lattice topologies that serve more effectively specific loading conditions. Huang et al. [32] investigated the use of the bidirectional evolutionary structural optimization (BESO) technique in order to find topologies that offer maximum bulk or shear modulus.

\subsection{Homogenization of lattice cells}

In order to reach an optimal solution, the search space has to be explored and the candidate solutions have to be evaluated on their quality regarding the stress field and displacement. The exact displacement and subsequently the stress field can be obtained by the solution of a differential equation governing the behavior of the part under the prescribed loading. However, for the majority of the real parts, the solution of such an equation is practically impossible due to their geometrical complexity. The fact that more than one candidate solutions have to be evaluated increases the time and cost requirements even more. As a result, a numerical approach is preferred at most cases, which allows to decompose the part domain into simpler sub-domains and numerically solve a set of simpler equations [33]. For structural problems, the numerical method that has prevailed, among others such as the finite difference method and the boundary element method, is the finite element method (FEM) [34]. Therefore, FEA has become an essential step of every design optimization procedure. In the numerical analysis of parts with lattice structures, two different approaches can be distinguished, namely the micro-scale and the macro-scale approach [7]. In the first approach, the exact lattice structure's geometry is modelled. However, this approach has an impact on the computational cost of the analysis, making it a hard task even for modern computers, since the geometry is very complex [35], [36]. The repeated evaluations of the objective function needed in a design optimization problem make this task even harder [37]. On the other hand, as the lattice geometry is periodic, each cell can be modelled as a volume of a homogenous material, the so called representative volume element (RVE), resulting in a macro-scale analysis and therefore reduced computational cost. The new homogenous material must have mechanical properties that will give the same response as the lattice cell. These properties are called effective material properties and can be either isotropic or orthotropic, depending on the lattice cell topology and dimensional parameters. Finding these properties requires the application of a technique known as "homogenization". Both analytical and computational homogenization methodologies can be found in the literature. Analytical homogenization methods utilize classical solid mechanics theories, such as the Euler-Bernoulli and Timoshenko beam theories and the Hill's anisotropic yield criterion, in order to obtain the equivalent elastic and shear moduli, Poisson's ratio and yield surfaces for the unit cell [7], [38]-[41]. However, applying analytical methods to homogenize unit cells of complex topology is impractical and can become even infeasible as the complexity of the cell topology increases. In order to overcome this obstacle, computational homogenization methodologies 
have been developed. Nguyen and Noels [36] presented a second-order computational homogenization framework for cellular materials aiming to capture accurately the buckling effects by using the discontinuous Galerkin (DG) method to formulate the problem. Dirrenberger et al. [42] used FEA to obtain the elastic moduli of two-dimensional and three-dimensional auxetic lattice cells. A very good agreement between FEA results and analytical expressions for the elastic moduli and the yield surfaces was found by Deshpande et al. [38] when studying the mechanical properties of the octet-truss lattice cell. Good agreement between finite element, analytical and also experimental results was found by Chen et al. for the homogenized properties of a novel anti-tetrachiral anisotropic lattice structure [39]. Vigliotti et al. [43] presented a methodology for accounting non-linear material behavior when performing numerical homogenization of lattice cells. The methodology was applied in the homogenization of hexagonal and triangulated cells. Numerical results from models using the homogenized properties showed good agreement with results from models with explicit modelling of the lattice structures. Karamooz Ravari et al. [44] investigated the mechanical properties of lattice structures fabricated with fused deposition modelling (FDM). Their findings indicate that in order to obtain accurate mechanical properties, the variation of the strut diameter along its axis, caused by the FDM, has to be modelled. The effect of FDM process parameters in the effective mechanical properties of cubic, dode and diamond unit cells, was investigated by Park et al. [45]. Labeas and Ptochos calculated the Elastic modulus and Poisson's ratio of micro-lattice cellular structures and used the the derived elasticity matrices for the homogenisation of numerical models [46], [47].

\subsection{Research gap}

From the literature review, a research gap in the design optimization for lattice structures can be observed. Various optimization approaches, ranging from empirical ones to typical topology optimization methods such as BESO, have been proposed. However, there is no approach utilizing well-established multipurpose optimization algorithms, so that it ends up being an easy to validate and integrate software tool for design of lattice parts. In addition, the proposed homogenization methods for lattice cells are characterized by enormous complexity and require deep knowledge in the field of mechanics of materials, being inaccessible to the average industry practitioner.

\subsection{Scope of this study}

This paper aims to present a user-friendly computational framework for optimizing the design of parts which feature lattice structures. The aim of the optimization is to determine the optimal strut diameter value for each lattice cell in order to minimize the weight of the part, without exceeding a certain displacement value under a prescribed loading. Initially, a FEA homogenization of the lattice cell is performed for the lattice topology and the material used. In the next step, the part's geometry is modelled 
using RVEs for representing the lattice structure so that the computational cost of the analysis is reduced. Then, with the use of a genetic algorithm (GA), an optimal solution of the strut diameter value for each cell is obtained.

The structure of the paper is organized as follows. Section 2 presents the proposed methodology. The homogenization and design optimization strategies are described in detail. In Section 3, the cell topology used in this study is presented and the homogenized properties are derived through FEA. The part geometry, the GA's parameters and the results of the optimization are presented in Section 4. Finally, the findings of this study are summarized in Section 5 and the potential for further research is presented.

\section{Method}

\subsection{Proposed workflow}

The workflow proposed in this study consists of three phases (i.e. pre-processing, processing and postprocessing) as illustrated in Figure 2. In the pre-processing phase, the user initially selects a lattice topology and the regions of the part that will be replaced by lattice cells, based on empirical observations or previous knowledge for the specific application. Subsequently, the homogenization of the lattice cell, in order to obtain its effective mechanical properties, is performed numerically with the use of FEA. For that purpose, the cell topology, the dimensional parameters that will be optimized and the material properties are defined. The validation of the homogenization results can be performed either experimentally or by comparison with existing homogenized properties found in the literature for the cell topology studied. After obtaining the effective material properties of the lattice cell, the lattice design optimization is performed. The part geometry, the lattice regions and the design constraints are defined into the GA-FEA environment. In order to use the GA for obtaining the solution of the optimization problem, the algorithm's parameters must be defined as well. In the processing stage, the GA generates candidate solutions which are evaluated by FEA with respect to the displacement constraint. A solution, containing the optimal dimensional parameters of the unit cells, is obtained when the algorithm converges according to the convergence criterion set in the pre-processing stage. When processing has been completed and a solution has been obtained, a considerable amount of data, both from the GA and the FEA tool, has been generated. Therefore, a need for retrieving, storing and visualizing only the data that is of interest to the user exists. The data that is kept in the post-processing stage includes the optimized weight of the part, the maximum displacement of the optimal solution and computational metrics (i.e. computational time, number of generations, best and mean value at each generation) regarding the GA. The data related to the performance of the part (i.e. weight and displacement) can be used in order to 
validate the effectiveness of the method in a real case study by comparison with the actual part and its performance.

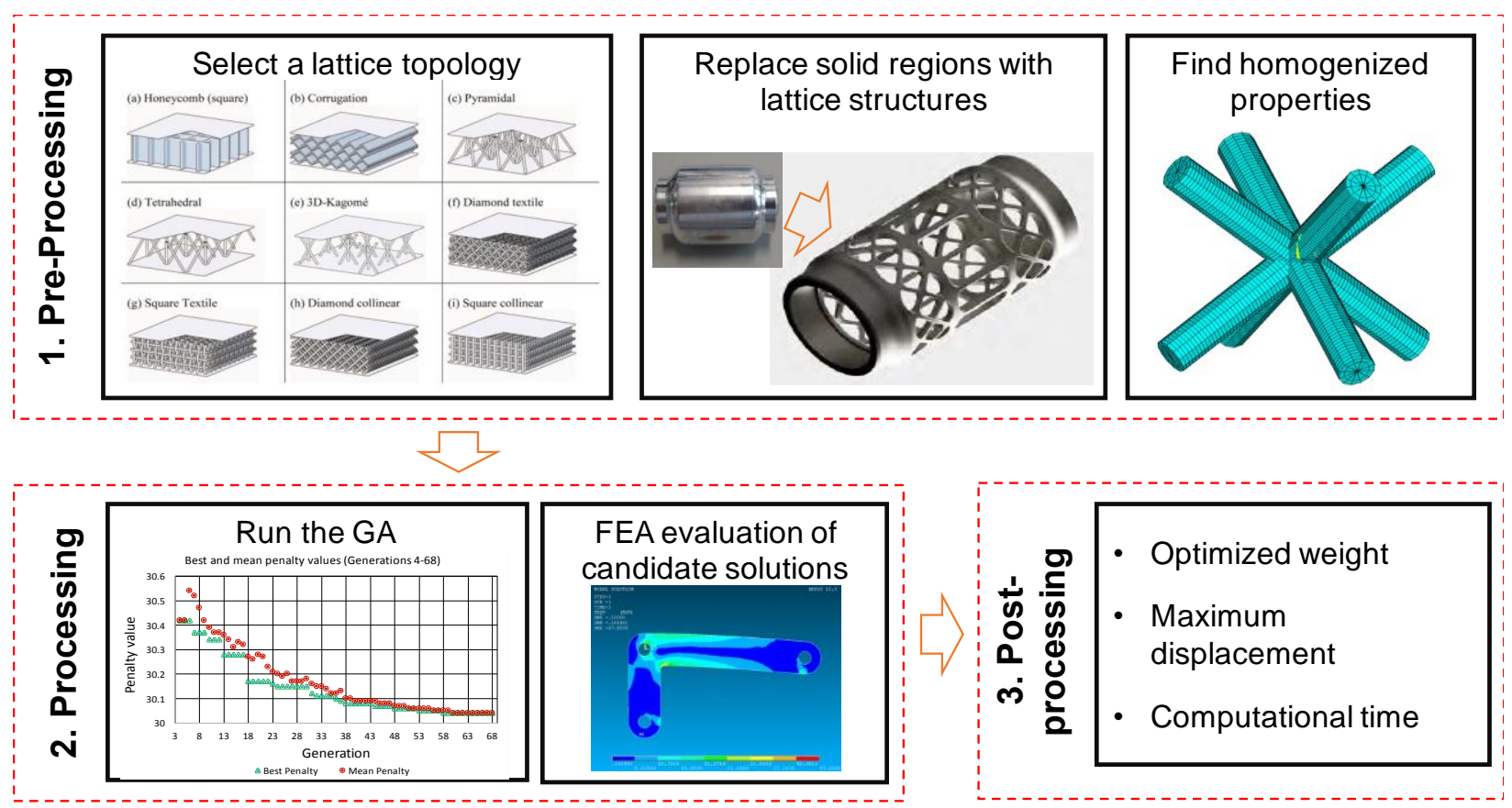

Figure 2. Schematic of the proposed workflow.

\subsection{Homogenization}

The homogenized properties of the unit cell are obtained by means of FEA. Only elastic properties are considered, in order to simplify the procedure of finding the effective mechanical properties of the unit cell. However, it should be stated that this assumption is acceptable when it is a priori known that the magnitude of the stresses in the cell is probably not going to cause plastic deformation. Therefore, this approach is not suitable when going into a more in-depth analysis, where elasto-plasticity is taken into account. The mechanical properties which are to be determined are the Young's modulus $\left(E_{i}\right)$ and the Poisson's ratio $\left(v_{i j}\right)$ in every direction. These two mechanical properties are presented hereafter in the context of the present analysis. The Young's modulus of a cell, $E_{i}$, in the direction $i$ can be obtained by the linear elasticity equation:

$$
E_{i}=\frac{\sigma_{i}}{\varepsilon_{i}}
$$

where $\sigma_{i}$ is the stress in the direction $i$ and $\varepsilon_{i}$ is the strain in the same direction. If a cuboid RVE is considered, the stress in any direction is equal to the sum of the forces applied parallel to this direction divided by the area of the face normal to this direction. The strain in the same direction is equal to the 
displacement, as a result of the applied forces, divided by the original dimension of the RVE in this direction. The Poisson's ratio, $v_{i j}$, is calculated as the negative ratio of the transverse strain in the $j$ direction, $\varepsilon_{j}$, to the axial strain in the $i$ direction, $\varepsilon_{i}$ :

$$
v_{i j}=-\frac{\varepsilon_{j}}{\varepsilon_{i}}
$$

\subsection{Design optimization}

The problem discussed in this paper is determining the optimal strut diameter value $\left[d_{1}, d_{2}, \ldots, d_{k-1}, d_{k}\right]$ of each unit cell $i$ and for a part containing $k$ unit cells in total, in order to achieve minimum part weight. The strut diameter can take values between a lower bound, $L B$, and an upper bound, $U B$. In case the strut diameter can take only discrete values and is not a continuous variable, an integer constraint is also needed. This way, each discrete value can be mapped to an integer. In addition, the maximum absolute value of displacement shall not exceed a certain value, maxdis. Therefore, the problem can be formulated as following:

minimize $\quad R=\operatorname{Weight}\left(d_{1}, d_{2}, \ldots, d_{k-1}, d_{k}\right)$

subject to the constraints:

$$
\begin{aligned}
& L B \leq a_{i} \leq U B, \forall i \in[1, k] \\
& \text { maximum displacement } \leq \text { maxdis } \\
& a_{i} \text { integers }
\end{aligned}
$$

This optimization problem is non-linear, as the displacement field is governed by differential equations, and the solution space can become very large as the number of struts grows. As a result, finding the optimal solution with exhaustive search of the solution space can require enormously long computational time. Therefore, an intelligent search technique has to be applied. In this study, a GA is employed for the efficient search of the solution space. GAs, inspired from the natural selection process, find their roots in the mid 1970's [48] and are one of the most efficient methods for solving hard optimization problems [49]. GAs evaluate the quality of a candidate solution, called an individual, according to its fitness, which depends on how much the objective function is minimized for the specific solution and the extent at which the solution violates the constraints set. This extent is expressed by the value of the TotalPenalty parameter. Therefore, the fitness of a solution $\left[d_{1}, d_{2}, \ldots, d_{k-1}, d_{k}\right]$ can be expressed as following, since the problem investigated is a minimization one:

$$
\text { fitness }\left(d_{1}, d_{2}, \ldots, d_{k-1}, d_{k}\right)=\frac{1}{R}-\operatorname{TotalPenalty}\left(d_{1}, d_{2}, \ldots, d_{k-1}, d_{k}\right)
$$

The GA begins with a set of initial solutions which constitute the initial population. Each solution is represented by a chromosome which consists of a number of genes, with each gene carrying a numerical 
value [50]. The way that solutions are transformed to chromosome representations is called encoding. The reverse procedure, where chromosomes are transformed to solutions with the representation of the problem domain, is called decoding. As the algorithm progresses, certain solutions from one population are selected and modified suitably by the GA operators in order a new population, better than the previous one, is formed. The selection procedure is designed so that the best individuals from the current population (i.e. those with the largest fitness values) are more likely to be selected as the basis for the next generation. In the present case, FEA is used for investigating the constraint violation of a solution and thus determining the fitness of this solution. The next generation is built by two operators, namely the crossover operator and the mutation operator. The crossover operator selects genes from different parent chromosomes and combines them in order to produce a child chromosome belonging to the next generation. The mutation operator modifies the values of the genes inside child chromosomes in order to maintain genetic diversity and prevent the algorithm from getting stuck at a local optimum. A stopping criterion for the GA must also be established. Although many different stopping criteria can be found in the literature, some of the most common are limiting the available computational time, limiting the maximum number of generations or reaching a satisfactory value of the objective function.

\section{Finite element analysis of a unit cell}

The lattice cell studied in this paper follows the topology of a Body-Centered Cubic (BCC) unit cell, which means that it consists of 8 identical struts that start from each corner of the cell and are joined at its center. This particular lattice topology was selected due to its geometrical simplicity which makes it ideal for integrating in parts of almost any geometry and also for numerical modelling. The strut diameter, $d$, can take values between 0.4 and $0.8 \mathrm{~mm}$ in steps of $0.1 \mathrm{~mm}$. The selection of the lower and upper diameter bound and also the step value is deriving and is compliant to the dimensional accuracy capabilities of AM processes for metallic parts [51], [52]. The cube enclosing the lattice cell has an edge length, $l$, which is equal to $2.5 \mathrm{~mm}$. The edge length selection depends on the design characteristics of the developed component. For example, the thickness of the part and the number of cells that will be used through the thickness will determine the edge size of the lattice cell.

The weight of a unit cell $i$ is calculated by the following formula:

$$
w_{i}=\frac{\rho}{\rho_{0}} W
$$

Where, $\rho$ is the density of the unit cell, $\rho_{0}$ is the density of the solid material, and $W$ is the weight of an $l^{3}$ $\mathrm{mm}^{3}$ cube of full density and is equal to:

$$
W=\rho_{0} l^{3}
$$


The relative density, $\frac{\rho}{\rho_{0}}$, of the cell with respect to the solid material density, $\rho_{0}$, is calculated by the following formula:

$$
\frac{\rho}{\rho_{0}}=\left(\frac{d}{2 l}\right)^{2} 4 \pi \sqrt{3}
$$

The material used in this study is the titanium alloy Ti6Al4V. The material properties, presented in Table 1, are published by Renishaw for SLM processed Ti6Al4V [53].

Table 1. Ti6Al4V material properties.

\begin{tabular}{|l|l|}
\hline \multicolumn{1}{|c|}{ Physical property } & \multicolumn{1}{c|}{ Value } \\
\hline Young's modulus & $113.8 \mathrm{GPa}$ \\
\hline Poisson's ratio & 0.342 \\
\hline Density & $4420 \mathrm{~kg} / \mathrm{m}^{3}$ \\
\hline Yield strength $(0.2 \%)$ & $1070 \mathrm{MPa}$ \\
\hline
\end{tabular}

In order to find the homogenized material properties of the unit cell, a number of numerical simulations were performed. For the purpose of modelling the lattice cell, beam elements were preferred against solid elements because they have less nodes offering reduced analysis time and can also produce accurate results in studies involving truss structures. The lattice cell was modelled using ANSYS BEAM188 elements. This type of elements is based on the Timoshenko theory. Thus, it takes into account sheardeformation effects and can be used for thick beam structures (i.e. radius-to-length ratio greater than 0.1). For the present case the radius-to-length ratio is greater than 0.18 when the diameter is equal to $0.4 \mathrm{~mm}$ and therefore the use of such elements is recommended in order to ensure maximum accuracy. After a $1 \%$ displacement convergence study, each strut was divided in 40 sections along its axis and 12 sections along its circumference. The total elements the model contained were 320 in total. The mechanical properties of the cell are investigated only in one direction, as they are isotropic. A virtual compression test in order to derive the Young's modulus is performed. The compression test is performed in only one direction, since the cell carries isotropic effective properties. For that reason, a point force $F$ of $0.5 \mathrm{~N}$ parallel to the z-axis was applied in the nodes found in all the 8 edges of the cell. The force on the top nodes was in the opposite direction than that in the bottom nodes, in order to simulate compression. In that case, the compressive stress in the z-direction, $\sigma_{z}$ can be calculated as:

$$
\sigma_{z}=\frac{4 F}{l^{2}}
$$


The selection of the magnitude of the force is not affecting the results, as only the elastic properties of the material are taken into consideration. The center of the cell was constrained from all 6 degrees of freedom (DOFs) in order to prevent rigid body motion due to numerical instabilities. This does not have an impact on the results, since the loads and the dimensions of the struts are symmetric with respect to the center, hence the center practically has zero displacement under this loading. The meshed unit cell along with the loading conditions and the constraints is presented in Figure 3. An example of the computed displacements is presented in Figure 4, where the results for a cell with $0.4 \mathrm{~mm}$ strut diameter are presented. It can be observed, from the deformed shape, that the selected element type and meshing are capable of capturing the bending of the struts.

The effective Young's modulus in relation to the strut diameter derived from the FEA is presented in the graph of Figure 5. It can be observed that the Young's modulus takes values between 50 and $740 \mathrm{MPa}$, following an almost exponential trend. The effective Poisson's ratio in relation to the strut diameter is presented in Figure 6. Its values range from 0.482 to 0.492 and is also following an almost exponential trend.

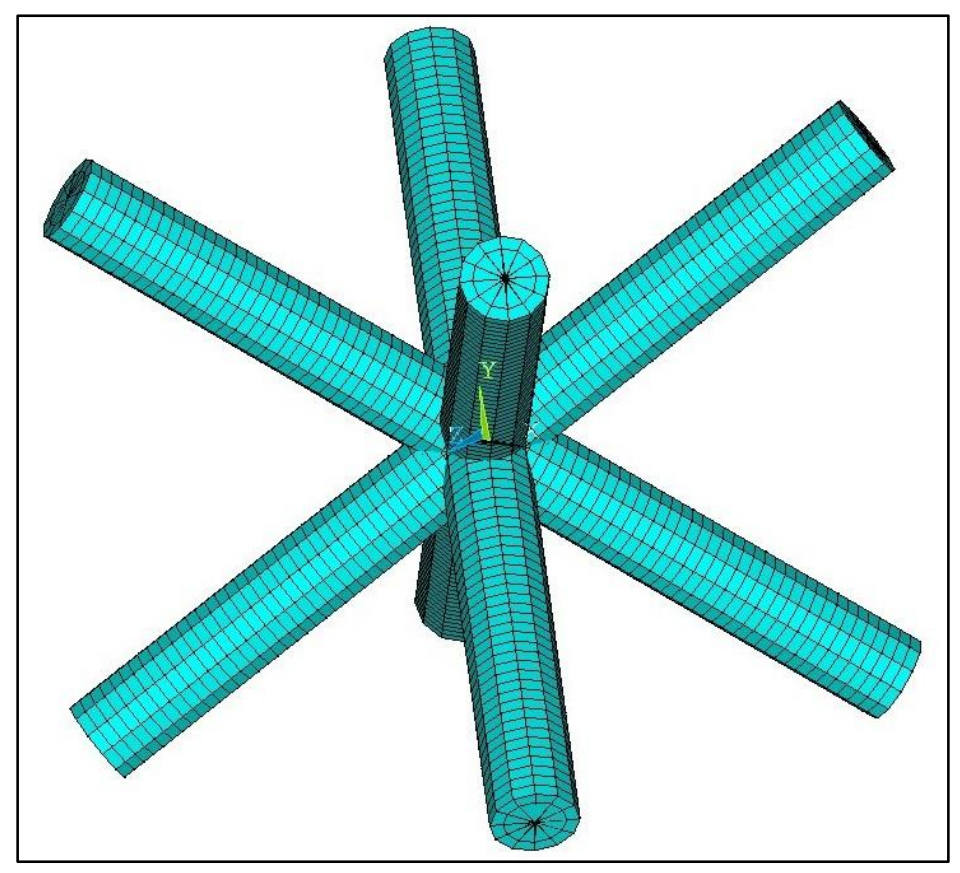

Figure 3. Finite element model of the unit cell. 


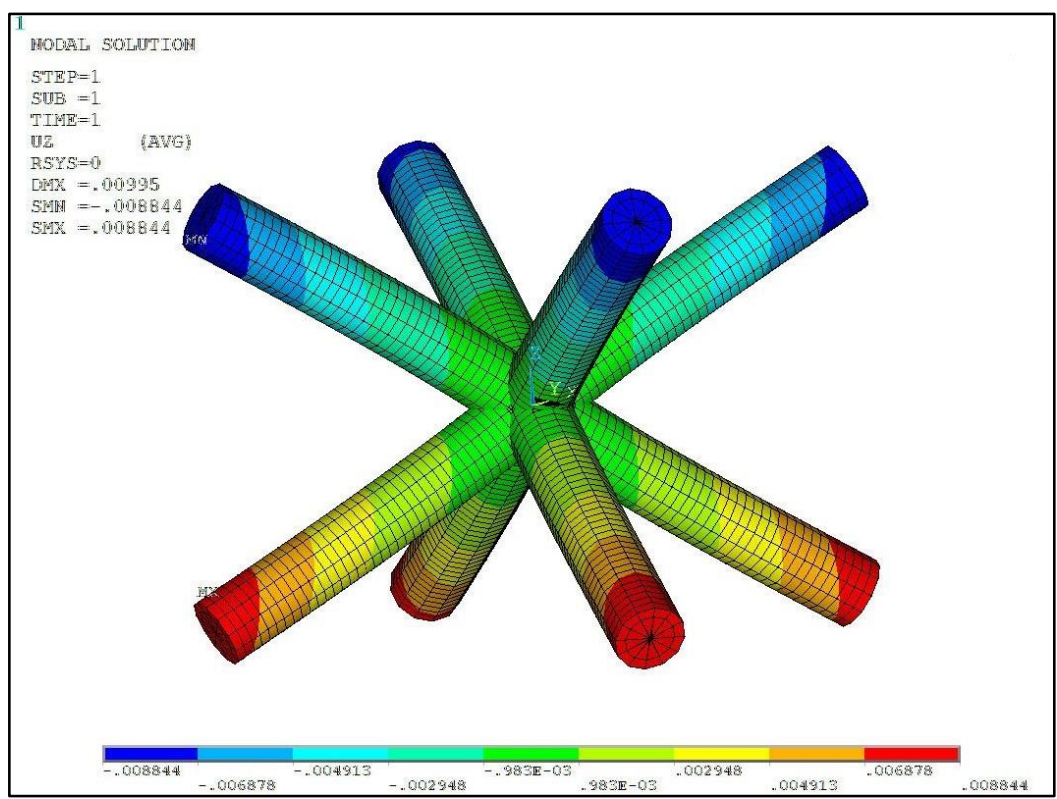

Figure 4. Displacement in the z-axis of a $0.2 \mathrm{~mm}$ strut diameter lattice cell.

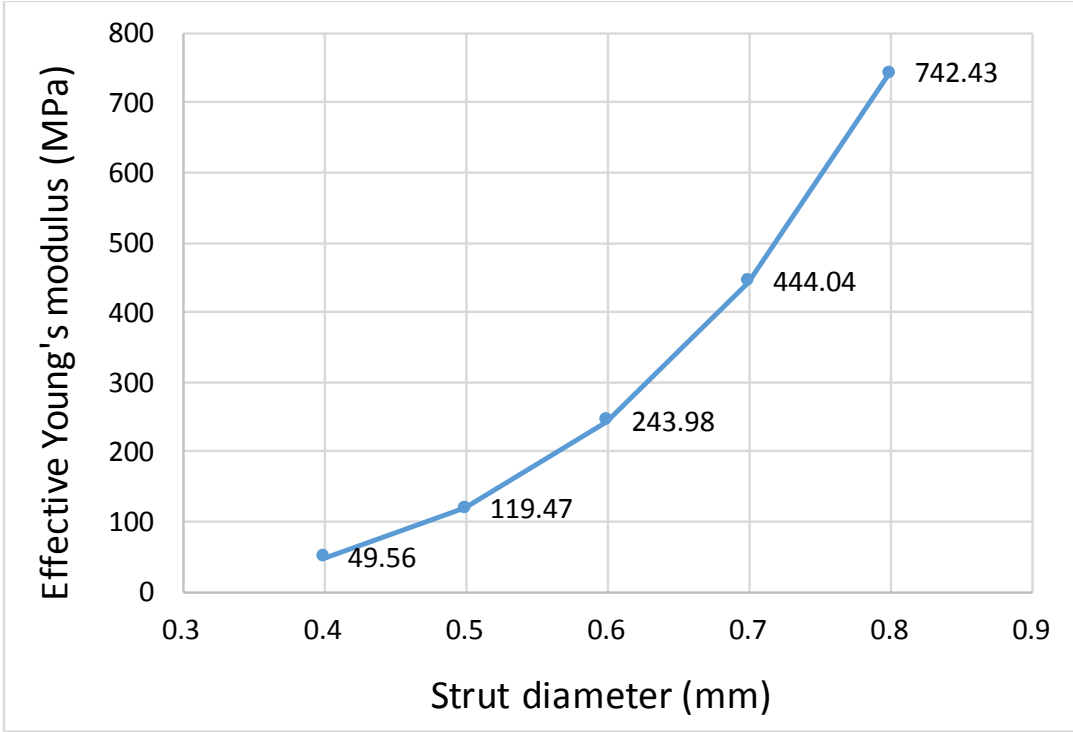

Figure 5. Unit cell effective Young's modulus. 


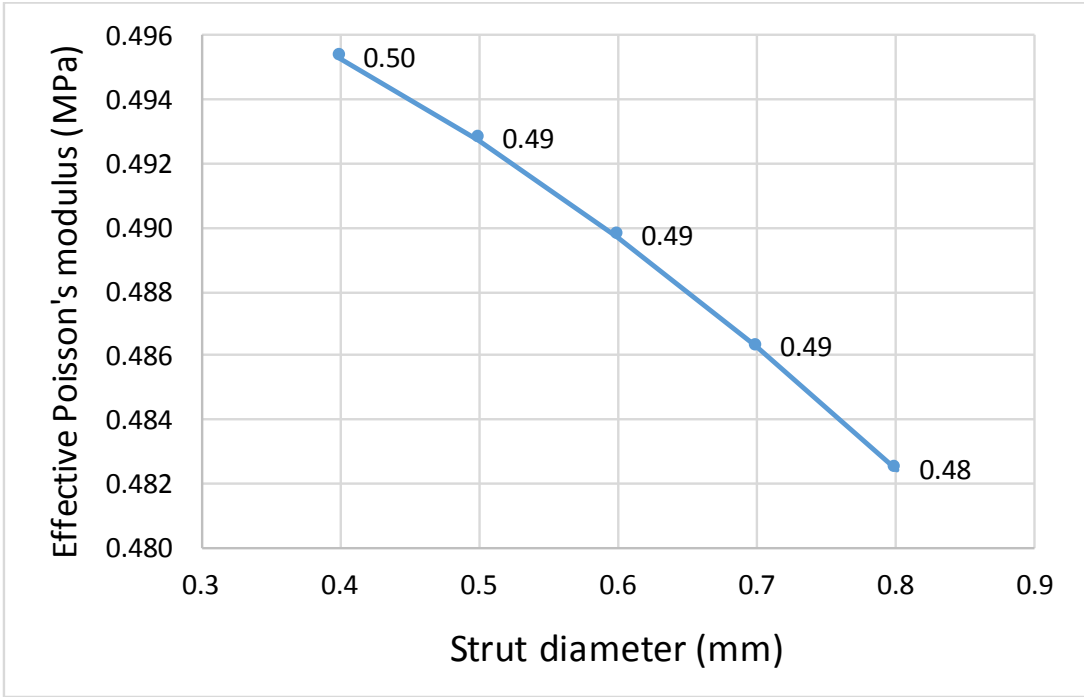

Figure 6. Unit cell effective Poisson's ratio.

\section{Design optimization case study}

\subsection{Part geometry and modelling}

For the validation of the proposed method, a simple case was developed. The geometry was deliberately chosen to be simple, as to allow for an understanding of the validity, effectiveness and computational requirements of the proposed workflow. The part to be optimized in this paper is a bracket made of Ti6A14V with dimensions as shown in Figure 7 and a thickness of $2.5 \mathrm{~mm}$, so as to fit one lattice cell through its thickness. The weight of the part consisting by solid material is equal to $34.737 \mathrm{~g}$. The bracket is subjected to a point force of $50 \mathrm{~N}$ parallel to the $\mathrm{y}$-axis and located below the top right hole and a point force of $50 \mathrm{~N}$ parallel to the $\mathrm{x}$-axis and located at the right of the bottom hole. In addition, the bracket is constrained from all DOFs at the internal surface of top left hole. The bracket geometry and dimensions were determined by trial-and-error so that it can withstand the prescribed loads without the von Mises stresses to surpass the material's yield strength, but without specific considerations about efficient distribution of the material. The reason is that this study aims to propose a design optimization methodology that can be applied to any initial design with no limitations to the geometry of the parts.

A preliminary analysis is performed with a bracket consisting only of solid material in order to investigate the stress distribution and the displacements. The bracket is modelled in ANSYS using tetrahedral SOLID285 elements. After a 4\% stress convergence study, the bracket was modelled with 7,907 elements in total, with the mesh getting finer at the holes' boundaries and the sharp corners. The meshed bracket with the applied loads is shown in Figure 8. The von Mises stress and displacement in the $\mathrm{x}$-axis fields are shown in Figure 9 and Figure 10 respectively. The maximum stress is observed in the corner between the 
longitudinal and transversal parts of the bracket and is almost equal to $48.081 \mathrm{MPa}$. The maximum absolute value of the displacement in the $\mathrm{x}$-axis is observed in the top rightmost region and is equal to $0.0213 \mathrm{~mm}$. Based on the stress distribution obtained, the regions of solid material to be replaced with lattice structures are determined as presented in Figure 11. The illustrated areas are those that have the lowest von Mises stresses compared with the rest of the part. Taking into account, that the lattice cells are behaving as a material with much less strength than the solid material, this selection is made so that it is ensured that the lattice structure will not collapse due to high stresses.

The bracket consisting of both solid material and lattice structures is then modelled. The lattice cells are modelled as RVEs using hexahedral SOLID185 elements. During the optimization, each lattice cell will have the material properties corresponding to its strut diameter as calculated previously. The solid material is modelled with SOLID285 elements as in the preliminary analysis. The model is presented in Figure 12, where the lattice regions are marked with purple color. A verification analysis using the material properties of the solid material in every region of the bracket is performed. The results are compared with the preliminary analysis, in order to verify that modelling certain regions with brick elements, instead of tetrahedral elements, has no or little effect in the results. As it can be observed from Figure 13, the stress distribution is the same as when the bracket is meshed with tetrahedral elements. In addition, the maximum von Mises stress is only $0.9 \%$ greater when using brick elements for the lattice regions. Therefore, it can be assumed that the effect of using brick elements for modelling the lattice regions is negligible.

In the present case, each lattice cell can have an independent value for the strut diameter, $d$, as described in Section 3. Since $d$ can only take discrete values, the optimization problem is an integer optimization problem. The variable representing the diameter can take an integer value between 1 and 5 with each value representing the real diameter value from 0.4 to 0.8 in steps of $0.1 \mathrm{~mm}$. The design constraint for the bracket is that the maximum displacement in the x-direction must not exceed the value of $0.072 \mathrm{~mm}$. In case all the cells have the minimum value for $d$, achieving the minimum possible weight, the maximum displacement in the $\mathrm{x}$-direction has been found to be $0.073 \mathrm{~mm}$. On the other hand, in the case that all the cells have the maximum values for $d$, resulting to the maximum possible weight, the maximum displacement in the $\mathrm{x}$-direction has been found to be $0.071 \mathrm{~mm}$. Therefore, the aforementioned design constraint is intentionally set in order to investigate the capability of the GA to effectively search the solution space and find a near-optimal solution. Since the total number of cells is 96 , the optimization problem can be expressed as:

minimize $\quad R=\sum_{i=1}^{96} W \operatorname{eight}\left(d_{1}, d_{2}, \ldots, d_{95}, d_{96}\right)$

subject to the constraints: 
$1 \leq d_{i} \leq 5, \forall i \in[1,96]$

maximum displacement $\leq 0.072$

$d_{1}, d_{2}, \ldots, d_{95}, d_{96}$ integers

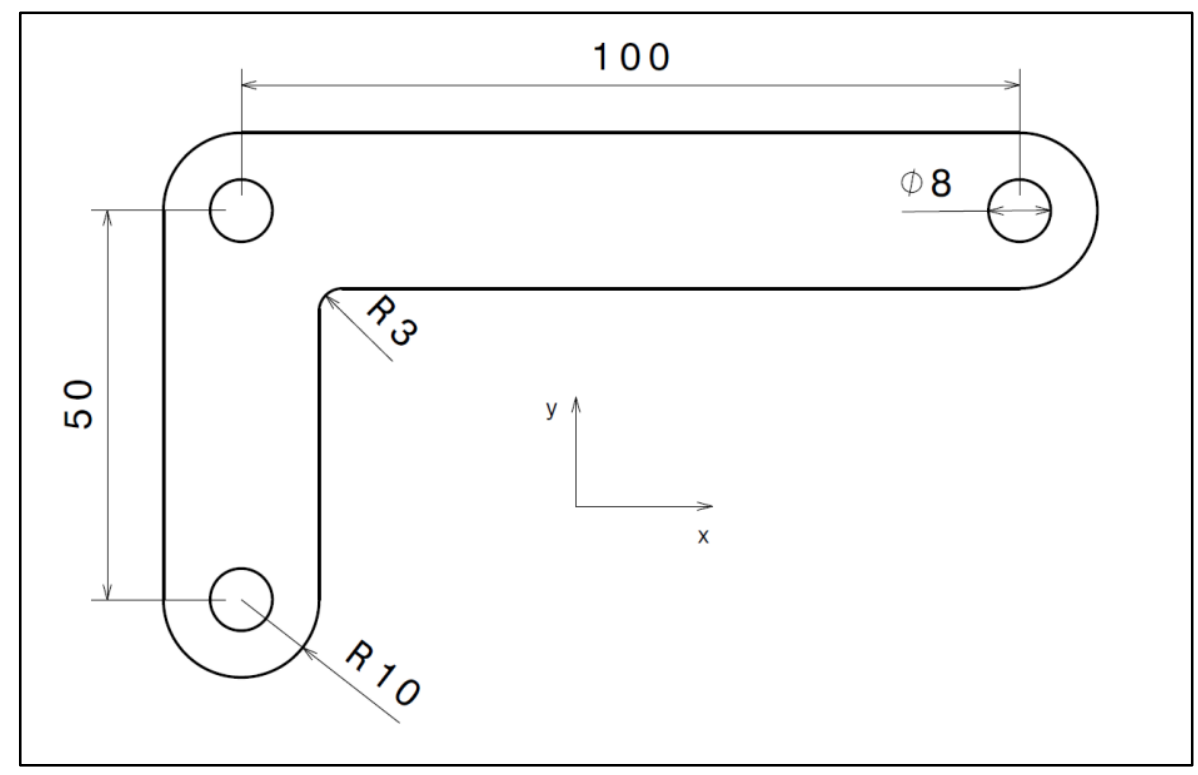

Figure 7. Bracket dimensions.

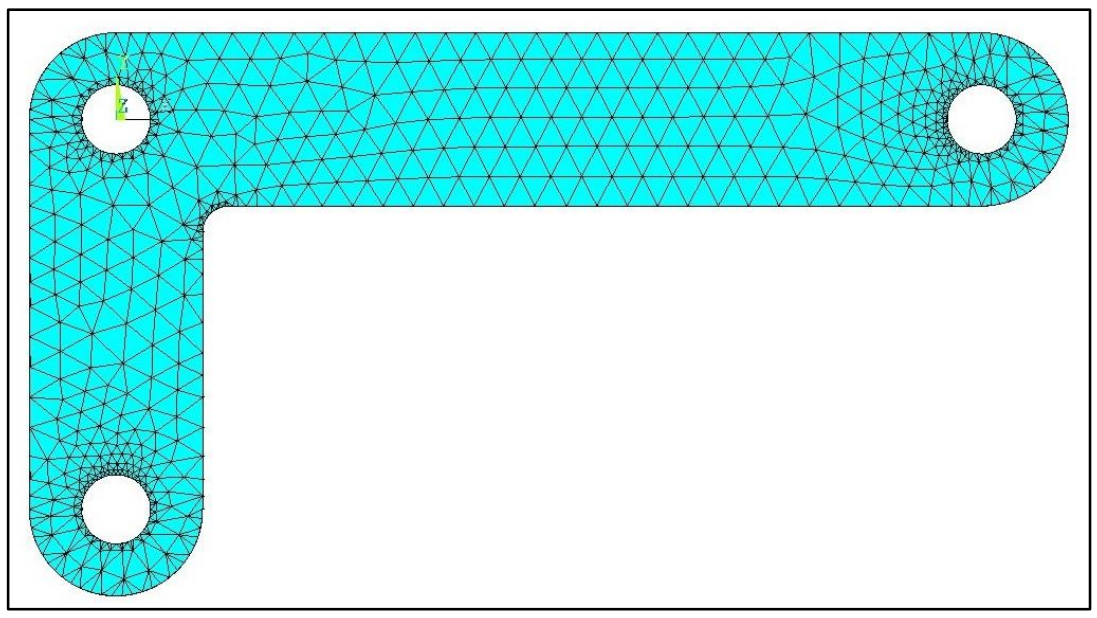

Figure 8. Finite element model of the solid bracket. 


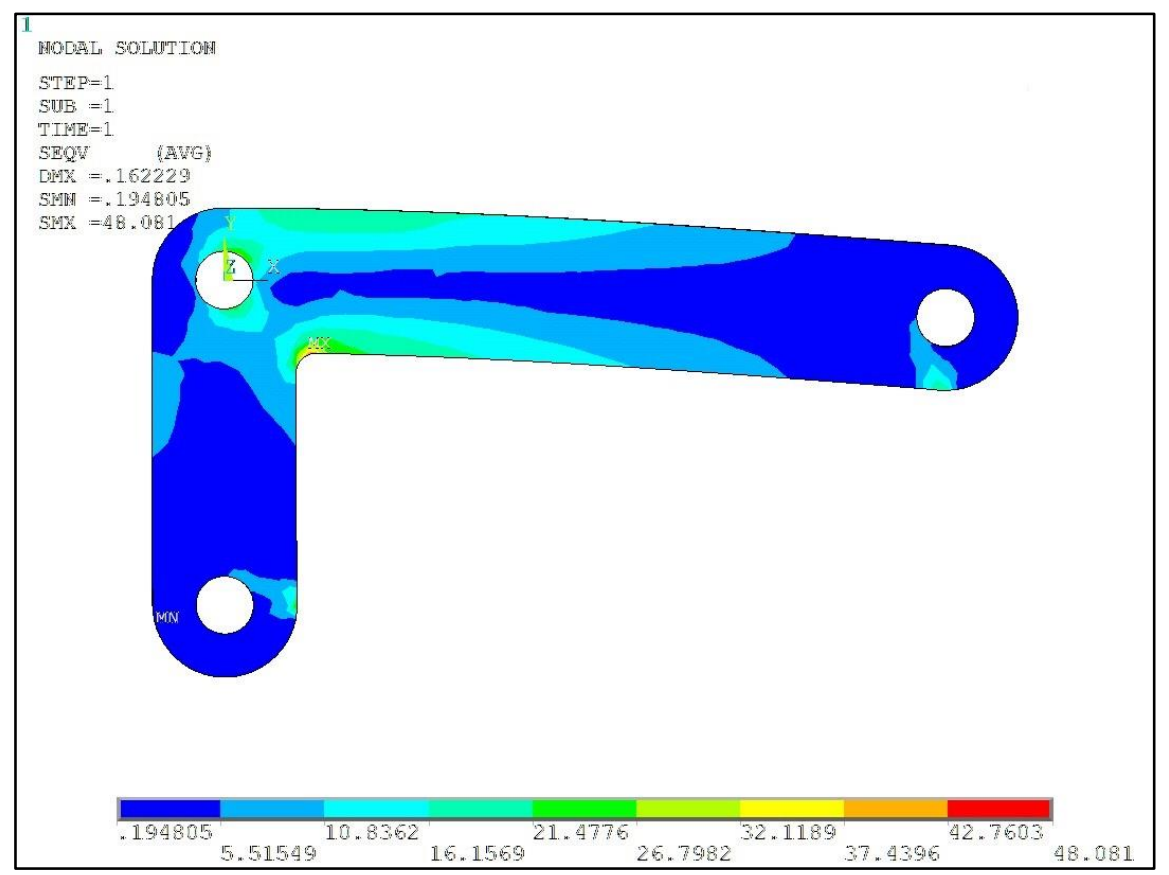

Figure 9. Von Mises stress results for the solid bracket.

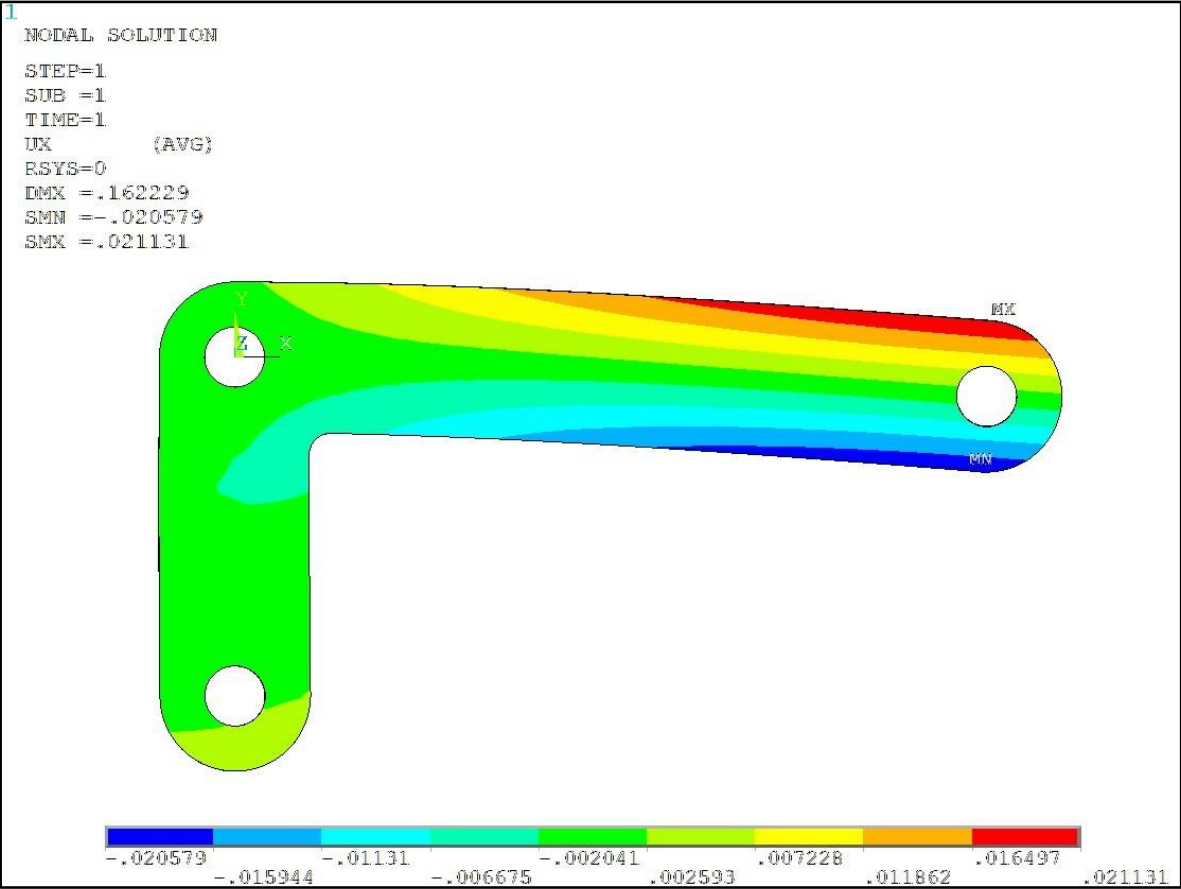

Figure 10. Displacement in the x-axis results for the solid bracket. 


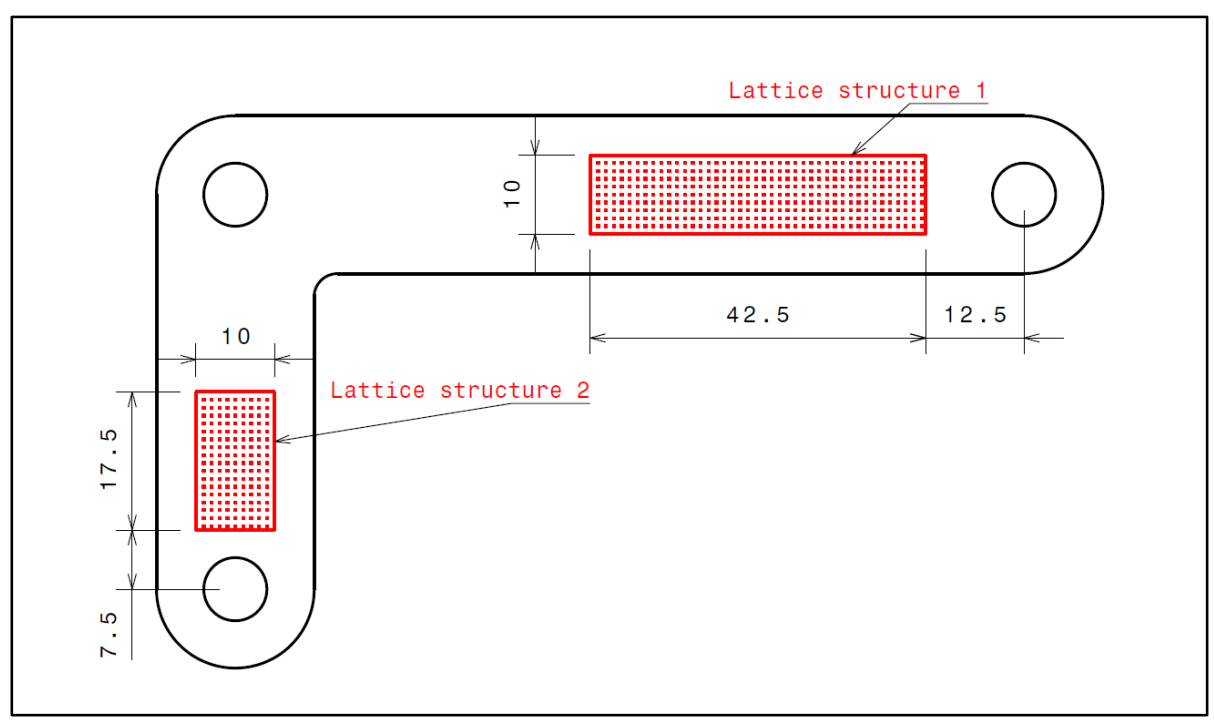

Figure 11. Lattice regions of the bracket.

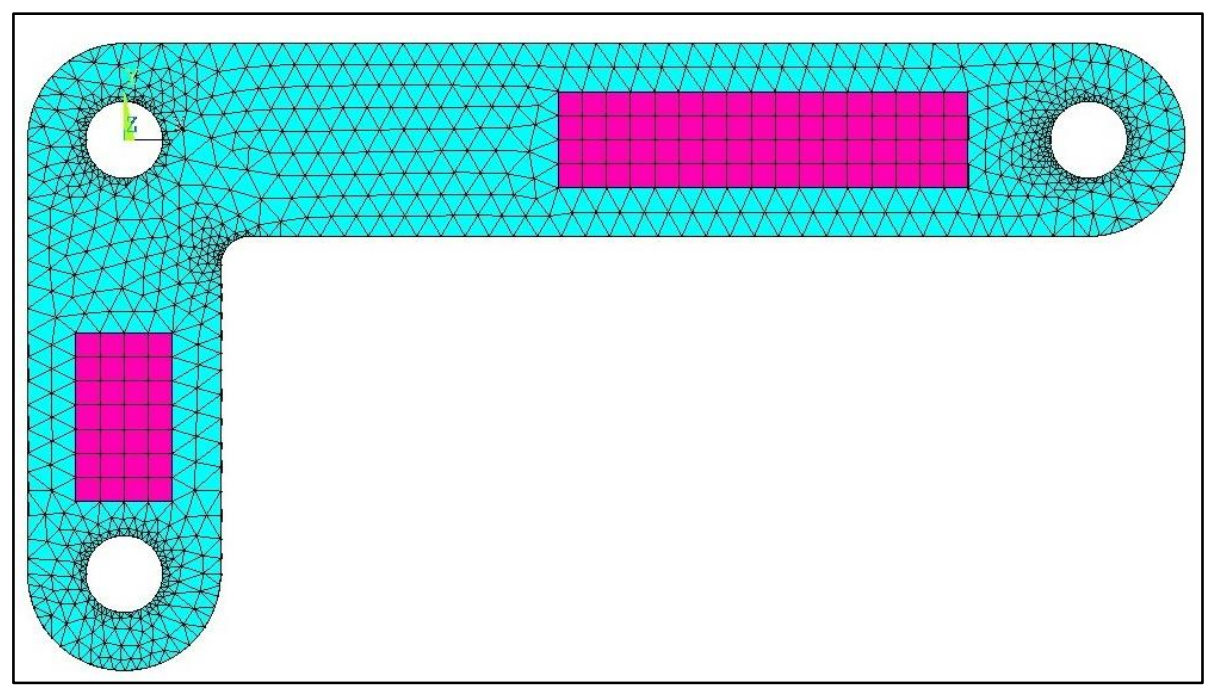

Figure 12. Modelling of the bracket using brick elements for the lattice structure. 


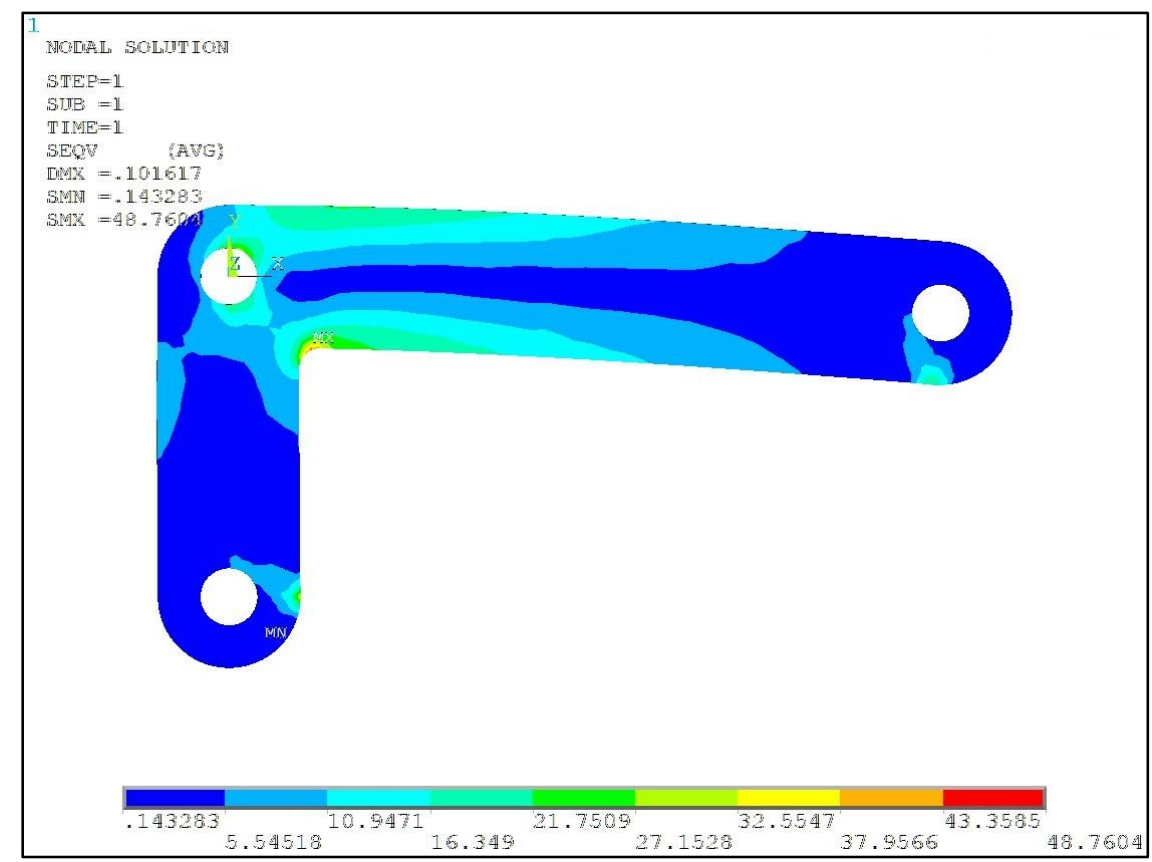

Figure 13. Von Mises stresses using brick elements for the lattice regions.

\subsection{Genetic algorithm}

The GA utilized in this study is the one that comes integrated in the MATLAB programming environment [54]. As GAs have been a research topic for more than 40 years [42], many different implementations can be found in the literature. The reasons for selecting the specific GA have mainly to do with userfriendliness. The MATLAB was chosen due to its strong pre- and post-processing capabilities and the simplicity of interfacing it with ANSYS, through a DOS prompt command. Furthermore, as the algorithm is ready to use out of the box, valuable developing time is saved and the user can focus on the engineering aspect of the optimization. On the other hand, the selection of the MATLAB GA comes with a remarkable trade-off. The algorithm does not allow the use of different selection, mutation and crossover operators than the already implemented. However, as the scope of this study is to propose a methodology for lattice design optimization, making the algorithm more efficient by using other operators is not investigated.

The selection function and the crossover and mutation operators of the algorithm are described in [55] and are designed so that the variables are enforced to take only integer values. Due to the presence of the integer constraint, the function optimized is not the objective function but the penalty function [54]. Selection of the best individuals in a population is performed using the tournament method. In this method, a number of tournaments are played between a number of individuals equal to $t$. The best individual from each tournament progresses to the mating pool (i.e. the set of individuals whose genes 
will be transferred to the next generation) in order to participate in the reproduction. If the selection is carried out systematically, each individual will participate in exactly $t$ tournaments. Thus, the overall best individual in the population will have $t$ copies of himself in the mating pool, while the worst individual will have no copies at all. For crossover, the Laplace methodology is used as described in [56], suitably modified to treat integer variables. The mutation operator is based on the mutation methodology described in [57], also modified to produce only integer solutions. A truncation procedure takes place after mutation and crossover in order to ensure that the variables satisfy the integer constraint. The variables that are not integer are truncated to one of the two nearest integer values with a probability equal to 0.5 . The algorithm handles the inequality constraints with a method described in [58].

The parameters of the algorithm are presented in Table 2. Since this study aims to show that the GA is capable of reaching a near-optimal solution for the specific problem, the parameters are set in such a way that the convergence of the algorithm is investigated. It should be stated that the GA belongs to the "random walk" algorithms and as a result the convergence criteria depend on the nature of the problem studied each time and can be set empirically or by using previous knowledge. In our case, convergence is considered to be achieved after 10 generations where the relative change in the best penalty value is lower than $10^{-6}$. Thus, the probability of being stuck in a local minima and assume that the algorithm has converged is limited. The population size for each generation was set equal to 10. Considering that for every individual a FEA is required to assess its penalty, the population size has a small value in order to reduce the computational time per generation. The number of generations and the time limit are infinite so that the algorithm does not stop before it converges.

Table 2. Genetic algorithm parameters.

\begin{tabular}{|l|l|}
\hline Parameter & Value \\
\hline Generations & Infinite \\
\hline Population size & 10 \\
\hline Stall generation limit & 10 \\
\hline Time limit & Infinite \\
\hline Tolerance & $10^{-6}$ \\
\hline
\end{tabular}




\subsection{Optimization results}

The algorithm was found to converge, according to the criteria described above, after 68 generations. The optimal weight was found to be $30.041 \mathrm{~g}$. The maximum displacement in the $\mathrm{x}$-direction was found to be 0.072 for the optimal solution, which means that the algorithm converged to the global optimum as the displacement is equal to the maximum allowed value. The time needed for the algorithm to converge was $2592 \mathrm{~s}$ on a dual-core Intel i5 computer, with the largest portion of the time used for the FEA runs.. The best and mean penalty value is illustrated for each generation.

In case that no feasible solutions have been generated by the algorithm, the penalty value of the infeasible solutions is equal to their constraint violation, according to how the MATLAB GA works [54]. For feasible solutions, the penalty value is equal to the objective function. This is the reason that while for the first 3 generations the penalty value has a value close to zero, then it suddenly rises to a value close to 30 . The first 3 generations contain only infeasible solutions, as illustrated in Figure 14 where the best and mean penalty values for each generation are shown. The value close to zero derives from infeasible solutions that violate the displacement constraint. The infeasibility of the solutions is caused by the randomness of the initial population. For a computationally optimized study, the initial population can be properly modified in order the algorithm to reach the feasible region more quickly. On the other hand, when the penalty value is close to 30 , it is obvious that we have feasible solutions, with the penalty value being equal to the weight of the part. The best and mean penalty values for the generations that produced feasible solutions are presented in Figure 15. It can be observed that the convergence of the algorithm starts at about 40 generations from the beginning of the optimization. Convergence can also be observed by the distance between the mean and the best fitness which is almost zero after the $60^{\text {th }}$ generation, meaning that the diversity of the population is very low and new solutions with significantly lower penalty values cannot be found. 


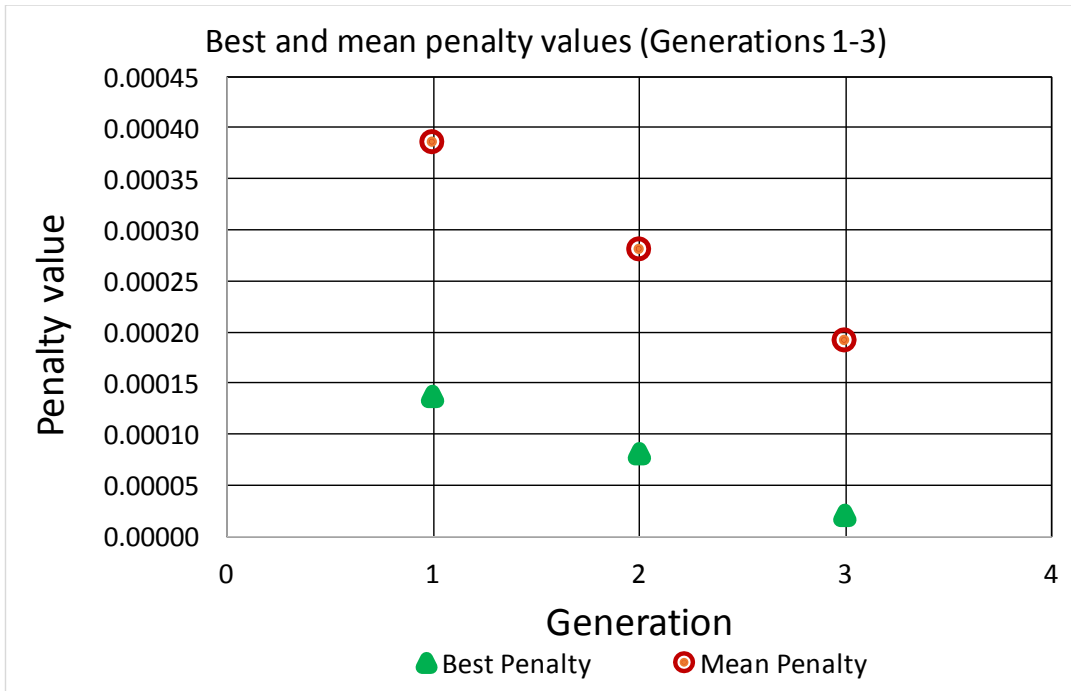

Figure 14. Best and mean penalty versus generations.

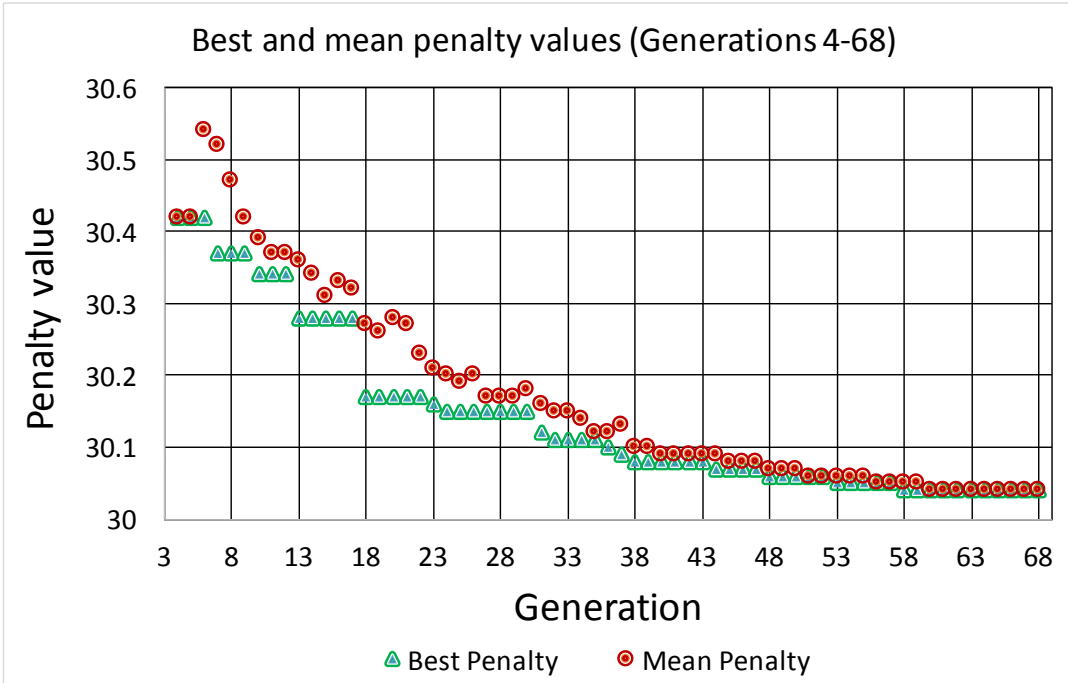

Figure 15. Best and mean penalty for the feasible region.

\subsection{Discussion}

The use of lattice structures can offer significant weight reductions in the final part. The weight of the part was decreased by almost $13.5 \%$ with the use of lattice structures. It has be shown that with the use of the GA for design optimization, the maximum displacement can be kept below a certain limit determined by the part mechanical performance requirements. In addition, it has been observed that homogenization can offer a time-efficient solution to the problem of modelling lattice structures. Nevertheless, the design optimization procedure is mostly based on an empirical approach by the user, since a robust methodology for replacing solid regions of the part with lattice structures has not yet been developed. 
From a computational perspective, the convergence of the GA to the optimal solution is slowed down by the fact that only infeasible solutions are generated in the first generations. As industrial needs dictate for time-efficient solutions and as the computational cost of the FEA is radically increasing for parts of larger scale, the convergence of the algorithm should be accelerated by modifying the initial population in order to contain one or more feasible solutions. Faster convergence will have a remarkable impact on the number of the necessary FEA runs for evaluation of all the candidate solutions' fitness. The effect of running both the GA and FEA from the same programming environment (e.g. both in MATLAB or both in APDL) on the total computational cost should be further investigated. However, the implementation of such a concept is not user-friendly since it requires much more amount of programming effort than the implementation presented in this study.

\section{Conclusions}

This paper demonstrates a novel methodology, which combines FEA with evolutionary optimization, for lightweight design of parts with lattice structures. Each lattice cell is replaced by a RVE, in order to reduce the computational cost. Simulation with the use of FEM is used in order to determine the effective mechanical properties (i.e. Young's modulus and Poisson's ratio) of the RVE with respect to the strut diameter. A GA is then utilized in order to find the optimal strut diameter for each cell of the lattice structure, in order to achieve minimum weight while not exceeding a certain value of the maximum displacement in the $\mathrm{x}$-axis.

The optimization results showed that the weight of the part is decreased by $13.5 \%$ when replacing certain regions of solid material with lattice structures. However, the maximum displacement was radically increased due to the lower elastic modulus of the lattice cells compared to the solid material. The maximum displacement can be held under a limit, determined by the performance requirements of the part, by implementing a design constraint in the GA. The use of the MATLAB GA has been proven capable of offering a user-friendly optimization environment, giving the opportunity to non-experts in the field of computer programming to implement the proposed workflow.

As the findings of this study show potential for further research, a number of topics for future work have been identified. From a computing perspective, future efforts will focus on investigating the effectiveness of optimization algorithms other than the GA, techniques to achieve faster convergence of the algorithms and also the integration of the optimization solver with the finite element solver into a single software tool. Furthermore, experimental validation is a strong prerequisite in order the aforementioned developments to become a robust tool for lattice design optimization. Experimental validation will be performed in two steps. In the first step, the finding of the lattice homogenization will be validated by 
compressive test of an actual lattice core. Secondly, the geometry of the case study will be fabricated by means of SLM and the optimal design found by the GA will be evaluated. Finally, as AM processes are constantly rising in popularity for the fabrication of lattice structures, the lattice optimization tool has to be supported by a library containing data about the lattice topologies and cell dimensional parameters that are feasible to be fabricated by AM processes and also models of the dimensional instabilities that are caused when building features at very small scales.

\section{References}

[1] European Commission, Analysis of options beyond 20\% GHG emission reductions: Member State results, $\quad$ Brussels, 2012, Available from: http://ec.europa.eu/clima/policies/package/docs/swd_2012_5_en.pdf.

[2] D. Chen, S. Heyer, S. Ibbotson, K. Salonitis, J.G. Steingrímsson, S. Thiede, Direct Digital Manufacturing: Definition, Evolution and Sustainability Implications, Journal of Cleaner Production 107 (2015) 615-625

[3] C. Emmelmann, P. Sander, J. Kranz, E. Wycisk, Laser Additive Manufacturing and Bionics: Redefining Lightweight Design, Phys. Procedia. 12, Part A (2011) 364-368.

[4] K. Salonitis K., S. Al Zarban, Redesign Optimization for Manufacturing Using Additive Layer Techniques, Procedia CIRP 36 (2015) 193-198, DOI: 10.1016/j.procir.2015.01.058

[5] K. Salonitis, Design for additive manufacturing based on the axiomatic design method", International Journal of Advanced Manufacturing Technology, (2016) published online (DOI: 10.1007/s00170016-8540-5)

[6] S.K. Moon, Y.E. Tan, J. Hwang, Y.-J. Yoon, Application of 3D printing technology for designing light-weight unmanned aerial vehicle wing structures, Int. J. Precis. Eng. Manuf. Technol. 1 (2015) 223-228.

[7] T. Liu, Z.C. Deng, T.J. Lu, Design optimization of truss-cored sandwiches with homogenization, Int. J. Solids Struct. 43 (2006) 7891-7918.

[8] W. van Grunsven, E. Hernandez-Nava, G. Reilly, R. Goodall, Fabrication and Mechanical Characterisation of Titanium Lattices with Graded Porosity, Metals (Basel). 4 (2014) 401-409.

[9] C. Yan, L. Hao, A. Hussein, S.L. Bubb, P. Young, D. Raymont, Evaluation of light-weight AlSi10Mg periodic cellular lattice structures fabricated via direct metal laser sintering, J. Mater. Process. Technol. 214 (2014) 856-864.

[10] C. Yan, L. Hao, A. Hussein, D. Raymont, Evaluations of cellular lattice structures manufactured using selective laser melting, Int. J. Mach. Tools Manuf. 62 (2012) 32-38. 
[11] P. Michaleris, Modeling metal deposition in heat transfer analyses of additive manufacturing processes, Finite Elem. Anal. Des. 86 (2014) 51-60.

[12] Y. Feng, G. McGuire, O. Shenderova, H. Ke, S. Burkett S. Fabrication of copper/carbon nanotube composite thin films by periodic pulse reverse electroplating using nanodiamond as a dispersing agent. Thin Solid Films. 2016.615: 116-121

[13] X. Su, Y. Yang, P. Yu, J. Sun, Development of porous medical implant scaffolds via laser additive manufacturing, Trans. Nonferrous Met. Soc. China. 22, Supple (2012) s181-s187.

[14] R.A.W. Mines, S. Tsopanos, Y. Shen, R. Hasan, S.T. McKown, Drop weight impact behaviour of sandwich panels with metallic micro lattice cores, Int. J. Impact Eng. 60 (2013) 120-132.

[15] B. Schoinochoritis, D. Chantzis, K. Salonitis, Simulation of metallic powder bed additive manufacturing processes with the finite element method: A critical review, Proc. Inst. Mech. Eng. Part B J. Eng. Manuf. (2015), In press, DOI: 10.1177/0954405414567522.

[16] M. Jordan, Y. Feng, S. Burkett, Development of seed layer for electrodeposition of copper on carbon nanotube bundles. Journal of Vacuum Science \& Technology B. 2015.33(2): 021202

[17] L. D’Alvise L., D. Chantzis, B. Schoinochoritis, K. Salonitis K., Modeling of part distortion due to residual stresses relaxation: An aeronautical case study, Procedia CIRP 31 (2015) 447 - 452, DOI: 10.1016/j.procir.2015.03.069.

[18] K. Salonitis, L. D’Alvise, B. Schoinochoritis, D. Chantzis, Additive manufacturing and postprocessing simulation: laser cladding followed by high speed machining, International Journal of Advanced Manufacturing Technology 85 (2016) 2401-2411

[19] V.J. Challis, X. Xu, L.C. Zhang, A.P. Roberts, J.F. Grotowski, T.B. Sercombe, High Specific Strength and Stiffness Structures Produced Using Selective Laser Melting, Mater. Des. 63 (2014) 783-788.

[20] T. Niendorf, F. Brenne, M. Schaper, Lattice Structures Manufactured by SLM: On the Effect of Geometrical Dimensions on Microstructure Evolution During Processing, Metall. Mater. Trans. B. 45 (2014) 1181-1185.

[21] O. Cansizoglu, O. Harrysson, D. Cormier, H. West, T. Mahale, Properties of Ti-6Al-4V nonstochastic lattice structures fabricated via electron beam melting, Mater. Sci. Eng. A. 492 (2008) 468474.

[22] J. Karlsson, A. Snis, H. Engqvist, J. Lausmaa, Characterization and comparison of materials produced by Electron Beam Melting (EBM) of two different Ti-6Al-4V powder fractions, J. Mater. Process. Technol. 213 (2013) 2109-2118. 
[23] A. Bauereiß, T. Scharowsky, C. Körner, Defect generation and propagation mechanism during additive manufacturing by selective beam melting, J. Mater. Process. Technol. 214 (2014) 24972504.

[24] J. Nguyen, S. Park, D. Rosen, Heuristic optimization method for cellular structure design of light weight components, Int. J. Precis. Eng. Manuf. 14 (2013) 1071-1078.

[25] G. Reinhart, S. Teufelhart, Load-adapted design of generative manufactured lattice structures, Phys. Procedia. 12 (2011) 385-392.

[26] G. Reinhart, S. Teufelhart, Optimization of mechanical loaded lattice structures by orientating their struts along the flux of force, Procedia CIRP. 12 (2013) 175-180.

[27] X. Huang, Y.M. Xie, Optimal design of periodic structures using evolutionary topology optimization, Struct. Multidiscip. Optim. 36 (2008) 597-606.

[28] B. Niu, J. Yan, G. Cheng, Optimum structure with homogeneous optimum cellular material for maximum fundamental frequency, Struct. Multidiscip. Optim. 39 (2009) 115-132.

[29] J. Chu, S. Engelbrecht, G. Graf, D.W. Rosen, A comparison of synthesis methods for cellular structures with application to additive manufacturing, Rapid Prototyp. J. 16 (2010) 275-283.

[30] L. Liu, J. Yan, G. Cheng, Optimum structure with homogeneous optimum truss-like material, Comput. Struct. 86 (2008) 1417-1425.

[31] X.J. Gu, J.H. Zhu, W.H. Zhang, The lattice structure configuration design for stereolithography investment casting pattern using topology optimization, Rapid Prototyp. J. 18 (2012) 353-361.

[32] X. Huang, a. Radman, Y.M. Xie, Topological design of microstructures of cellular materials for maximum bulk or shear modulus, Comput. Mater. Sci. 50 (2011) 1861-1870.

[33] E. Madenci, I. Guven, The Finite Element Method and Applications in Engineering Using Ansys, first ed., Springer Science + Business Media, New York, 2006.

[34] I. Ashcroft, A. Mubashar, Numerical Approach: Finite Element Analysis, in: L.M. da Silva, A. Öchsner, R. Adams (Eds.), Handbook of Adhesion Technology, Springer, Heidelberg, 2011: pp. 629660.

[35] S. Arabnejad, D. Pasini, Mechanical properties of lattice materials via asymptotic homogenization and comparison with alternative homogenization methods, Int. J. Mech. Sci. 77 (2013) 249-262.

[36] V.D. Nguyen, L. Noels, Computational homogenization of cellular materials, Int. J. Solids Struct. 51 (2014) 2183-2203.

[37] H.-G. Kim, S. Park, M. Cho, Structural topology optimization based on system condensation, Finite Elem. Anal. Des. 92 (2014) 26-35. 
[38] V.S. Deshpande, N. a. Fleck, M.F. Ashby, Effective properties of the octet-truss lattice material, J. Mech. Phys. Solids. 49 (2001) 1747-1769.

[39] Y.J. Chen, F. Scarpa, Y.J. Liu, J.S. Leng, Elasticity of anti-tetrachiral anisotropic lattices, Int. J. Solids Struct. 50 (2013) 996-1004.

[40] S.N. Khaderi, V.S. Deshpande, N. A. Fleck, The stiffness and strength of the gyroid lattice, Int. J. Solids Struct. 51 (2014) 3866-3877.

[41] G.W. Kooistra, V.S. Deshpande, H.N.G. Wadley, Compressive behavior of age hardenable tetrahedral lattice truss structures made from aluminium, Acta Mater. 52 (2004) 4229-4237.

[42] J. Dirrenberger, S. Forest, D. Jeulin, Effective elastic properties of auxetic microstructures: Anisotropy and structural applications, Int. J. Mech. Mater. Des. 9 (2013) 21-33.

[43] A. Vigliotti, V.S. Deshpande, D. Pasini, Non linear constitutive models for lattice materials, J. Mech. Phys. Solids. 64 (2014) 44-60.

[44] M.R. Karamooz Ravari, M. Kadkhodaei, M. Badrossamay, R. Rezaei, Numerical Investigation on mechanical properties of cellular lattice structures fabricated by fused deposition modeling, Int. J. Mech. Sci. 88 (2014) 154-161.

[45] S.-I. Park, D.W. Rosen, S. Choi, C.E. Duty, Effective mechanical properties of lattice material fabricated by material extrusion additive manufacturing, Addit. Manuf. 1-4 (2014) 12-23.

[46] E. Ptochos, G. Labeas, Elastic modulus and Poisson's ratio determination of micro-lattice cellular structures by analytical, numerical and homogenisation methods, Journal of Sandwich Structures and Materials 14 (2012) 597-626.

[47] G. Labeas, E. Ptochos, Homogenization of selective laser melting cellular material for impact performance simulation, International Journal of Structural Integrity, 6 (2015) 439 - 450 (2015)

[48] J.H. Holland, Adaptation in natural and artificial systems: An introductory analysis with applications to biology, control, and artificial intelligence., first ed., The MIT Press, Cambridge, 1992.

[49] A. Kaveh, M. Shahrouzi, Optimal structural design family by genetic search and ant colony approach, Eng. Comput. 25 (2008) 268-288.

[50] M. Melanie, An introduction to genetic algorithms, fifth ed., The MIT Press, Cambridge, 1996.

[51] I. Gibson, D.W. Rosen, B. Stucker, Additive Manufacturing Technologies, first ed., Springer Science+Business Media, New York, 2010.

[52] Fraunhofer IPK, Generative Manufacturing Methods: Selective Laser Melting, Available from: http://www.ipk.fraunhofer.de/fileadmin/user_upload/IPK_FHG/publikationen/themenblaetter/ps_ft_s elective_laser_melting_en.pdf. 
[53] Renishaw plc, Data sheet : laser melting powder titanium alloy Ti6Al4V ( grade 23 ): Processed using AM250 with 200 W laser, Stone, 2014, Available from: http://resources.renishaw.com/en/download/\%28ceb096ac7429486d8e288d04678ea873\%29

[54] MATLAB, Global Optimization Toolbox, URL: http://www.mathworks.com/products/globaloptimization/, Date accessed: 09/04/2015.

[55] K. Deep, K.P. Singh, M.L. Kansal, C. Mohan, A real coded genetic algorithm for solving integer and mixed integer optimization problems, Appl. Math. Comput. 212 (2009) 505-518.

[56] K. Deep, M. Thakur, A new crossover operator for real coded genetic algorithms, Appl. Math. Comput. 188 (2007) 895-911.

[57] K. Deep, M. Thakur, A new mutation operator for real coded genetic algorithms, Appl. Math. Comput. 193 (2007) 211-230.

[58] K. Deb, An efficient constraint handling method for genetic algorithms, Comput. Methods Appl. Mech. Eng. 186 (2000) 311-338. 
2016-10-21

\section{A hybrid finite element analysis and} evolutionary computation method for the design of lightweight lattice components with optimized strut diameter

\section{Salonitis, Konstantinos}

Springer Verlag

Salonitis K, Chantzis D, Kappatos V, A hybrid finite element analysis and evolutionary computation method for the design of lightweight lattice components with optimized strut diameter, International Journal of Advanced Manufacturing Technology, Volume 90, Issue 9-12, pÿ2017, pp. 26892701

http://dx.doi.org/10.1007/s00170-016-9528-x

Downloaded from Cranfield Library Services E-Repository 